\title{
Reducing the beta-shift in domain wall fermion simulations
}

\author{
Alban Allkoçi* ${ }^{*}$ \\ Computer Science Section \\ Polytechnic University of Tirana \\ Mother Theresa Square \\ Tirana - Albania \\ E-mail: albanefie.upt.al

\section{Artan Boriçi} \\ Physics Department \\ University of Tirana \\ Blvd. King Zog I \\ Tirana - Albania \\ E-mail: boriciefshn.edu.al
}

\begin{abstract}
The beta-shift induced from dynamical domain wall quarks leads to increased roughness of the gauge field, thus reversing the effect of smoothing from the gauge action improvement. By exploiting the relation of overlap and domain wall fermions in greater detail,we propose an algorithm which reduces the beta-shift to the level of dynamical overlap fermions.
\end{abstract}

XXIIIrd International Symposium on Lattice Field Theory

25-30 July 2005

Trinity College, Dublin, Ireland

\footnotetext{
* Speaker.

The authors participation in the conference and part of this work are funded from the NATO grant EAP.RIG.981410.
} 


\section{Introduction}

Lattice QCD with chiral fermions, although computationally expensive, is the best formulation of QCD on the lattice. There are two chiral formulations: a) Domain wall fermions [1 3 ] and b) overlap fermions [2], which are closely related [4, 5].

In this work we focus on the use of domain wall type fermions for lattice QCD simulations. Recent dynamical simulations with such fermions revealed larger chiral symmetry breaking than expected [6]. It was shown that the effect of gauge action improvement is effectively canceled by the dynamical domain wall fermion. Since there is no such problem with dynamical overlap fermions one wonders if there is something wrong with domain wall fermion formulation.

But as mentioned above these formulations are closely related and therefore if anything is to be blamed it is the implementation of domain wall fermions. It is the purpose of this work to show that the state-of-the-art of the dynamical domain wall implementation is not well suited for the state-of-the-art simulation algorithms.

\section{Notations}

In this work we will use the truncated overlap fermions [7] which have better chiral properties than the standard domain wall fermions [8]. The corresponding 5-dimensional operator is given by the $N_{5} \times N_{5}$ blocked matrix:

$$
\mathscr{M}=\left(\begin{array}{cccc}
D_{W}-\mathbb{1} & \left(D_{W}+\mathbb{1}\right) P_{+} & & -m\left(D_{W}+\mathbb{1}\right) P_{-} \\
\left(D_{W}+\mathbb{1}\right) P_{-} & D_{W}-\mathbb{1} & \ddots & \\
& \ddots & \ddots & \left(D_{W}+\mathbb{1}\right) P_{+} \\
-m\left(D_{W}+\mathbb{1}\right) P_{+} & & \left(D_{W}+\mathbb{1}\right) P_{-} & D_{W}-\mathbb{1}
\end{array}\right)
$$

where the blocks are matrices defined on the 4-dimensional lattices using the negative mass WilsonDirac operator $D_{W}, N_{5}$ is the number of time slices along the fifth Euclidean dimension and $P_{ \pm}$are chiral projection operators. Note that standard domain wall fermions use off-diagonal blocks which omit $D_{W}$.

Let $\mathscr{M}_{1}$ be the same matrix as above but with the bare quark mass $m=1$. Then it can be shown that [4, 5]:

$$
\operatorname{det} \mathscr{M}_{1}^{-1} \mathscr{M}=\operatorname{det} D^{\left(N_{5}\right)}
$$

where

$$
D^{\left(N_{5}\right)}=\frac{1+m}{2} \mathbb{1}+\frac{1-m}{2} \gamma_{5} \frac{\mathbb{1}-T^{N_{5}}}{\mathbb{1}+T^{N_{5}}}
$$

with $T$ the transfer matrix along the fifth dimension given by:

$$
T=\frac{\mathbb{1}+H_{W}}{\mathbb{1}-H_{W}}, \quad H_{W}=\gamma_{5} D_{W}
$$

Note also that in the large $N_{5} \operatorname{limit} D^{\left(N_{5}\right)}$ approaches the Neuberger overlap operator [9]:

$$
D=\frac{1+m}{2} \mathbb{1}+\frac{1-m}{2} \gamma_{5} \operatorname{sign}\left(H_{W}\right)
$$




\section{The problem}

The standard way one deals with the fermion determinant is expressing it as a Gaussian integral over pseudofermion fields:

$$
\left|\operatorname{det} D^{\left(N_{5}\right)}\right|^{2}=\left|\operatorname{det} \mathscr{M}_{1}^{-1} \mathscr{M}\right|^{2}=\int\left[d \Phi^{*} d \Phi\right] e^{-\left\|\mathscr{M}^{-1} \mathscr{M}_{1} \Phi\right\|^{2}}
$$

The resulting effective fermion action is given by:

$$
S_{\mathrm{PF}}=\left\|\mathscr{M}^{-1} \mathscr{M}_{1} \Phi\right\|^{2}
$$

From equation 2.1 we expect the action to have one contribution from the $D^{\left(N_{5}\right)}$ operator and some extra terms:

$$
S_{\mathrm{PF}}=\left\|\left[D^{\left(N_{5}\right)}\right]^{-1} \chi_{1}\right\|^{2}+\sum_{i=2}^{N_{5}}\left\|C_{i} \chi_{i}\right\|^{2}
$$

where $\chi_{i}$ are pseudofermion fields and $C_{i}$ are four dimensional matrices which in general may depend on gauge fields. This form of the action will be explicitly calculated below in this paper.

We note the extra terms in the action and we ask whether they would contribute in the generation of gauge fields. As long as the simulation algorithm averages over a large ensemble of pseudofermion fields $\chi_{i}$ these terms will cancel to give the correct determinant. However, fermion algorithms typically construct molecular dynamics trajectories which keep the pseudofermion field fixed. This may cause the exploration of gauge field configurations which "feel" the extra dimension through the extra terms.

These extra pseudofermion terms may be regarded as "artefacts" of the algorithm which contribute via the renormalised coupling:

$$
\beta \rightarrow c_{1} \beta+\Delta \beta
$$

As observed by [6] the renormalisation is such that it drives the gauge field toward the Aoki parity broken phase which in turn causes the breaking of the chiral symmetry.

We believe that this effect should go away if the extra terms are not present in the action. A direct evidence for this is not provided here, but simulations with overlap fermions clearly show that such renormalisation effects are absent.

\section{The solution}

First, let us calculate $S_{\mathrm{PF}}$. Using algebraic manipulations as in [-] and the results of the Appendix we get:

$$
S_{\mathrm{PF}}=\left\|\mathscr{M}^{-1} \mathscr{M}_{1} \Phi\right\|^{2}=\left\|\mathscr{T}^{-1} \mathscr{T}_{1} P^{T} \Phi\right\|^{2}
$$

where $\mathscr{T}$ is given by:

$$
\mathscr{T}=\left(\begin{array}{cccc}
P_{+}-m P_{-} & -T & & \\
& \mathbb{1} & \ddots & \\
& & \ddots & -T \\
-T\left(P_{-}-m P_{+}\right) & & & \mathbb{1}
\end{array}\right)
$$


and $P$ is the permutation matrix:

$$
\left(\begin{array}{cccc}
P_{+} & P_{-} & & \\
& P_{+} & \ddots & \\
& & \ddots & P_{-} \\
P_{-} & & & P_{+}
\end{array}\right)
$$

It is straightforward to show that (see Appendix):

$$
\mathscr{T}^{-1} \mathscr{T}_{1}=\left(\begin{array}{cccc}
{\left[D^{\left(N_{5}\right)}\right]^{-1}} & & & \\
B^{(2)} & \mathbb{1} & & \\
\vdots & & & \\
& & \ddots & \\
B^{\left(N_{5}\right)} & & & \mathbb{1}
\end{array}\right)
$$

where $B^{(i)}=T^{N_{5}-i+1}\left\{\left(P_{-}-m P_{+}\right)\left[D^{\left(N_{5}\right)}\right]^{-1}+\gamma_{5} \mathbb{1}\right\}, i=2, \ldots, N_{5}$. This way we get:

$$
S_{\mathrm{PF}}=\left\|\left[D^{\left(N_{5}\right)}\right]^{-1} \hat{\Phi}_{1}\right\|^{2}+\sum_{i=2}^{N_{5}}\left\|B^{(i)} \hat{\Phi}_{1}+\hat{\Phi}_{i}\right\|^{2}
$$

where $\hat{\Phi}_{1}=P_{+} \Phi_{1}+P_{-} \Phi_{N_{5}}$ and $\hat{\Phi}_{i}=P_{+} \Phi_{i}+P_{-} \Phi_{i-1}, i=2, \ldots, N_{5}$. It is easy to see that the second term of the right hand side constitutes the bulk of the coupling constant renormalisation.

This result hints to the following solution of the problem: express the fermion determinant in such a way that there are no additional terms in the effective fermion action. An obvious solution is to define:

$$
S_{\mathrm{PF}}=\left\|\left[D^{\left(N_{5}\right)}\right]^{-1} \phi\right\|^{2}
$$

where $\phi$ is now the usual pseudofermion field defined on the four dimensional lattice. The difficulty with this form is that the differentiation of (2.2) w.r.t. gauge field may yield numerically unstable expressions and ill-conditioned matrices.

A stable implementation may be defined as in the following: let $\varepsilon_{1}$ be defined as the blocked unit direction along the fifth dimension, i.e.,

$$
\varepsilon_{1}=(\mathbb{1}, 0, \ldots, 0)^{T}
$$

Using (4.1) we can write for the fermion determinant:

$$
\left|\operatorname{det} D^{\left(N_{5}\right)}\right|^{2}=\left|\operatorname{det} \varepsilon_{1}^{T} \mathscr{T}_{1}^{-1} \mathscr{T} \varepsilon_{1}\right|^{2}=\left|\operatorname{det} \varepsilon_{1}^{T} P^{T} \mathscr{M}_{1}^{-1} \mathscr{M} P \varepsilon_{1}\right|^{2}
$$

This expression can be used as a starting point to formulate a simulation algorithm in terms of numerically stable derivatives of $\mathscr{M}$-matrices. Hence, a variation of $D^{\left(N_{5}\right)}$ can be computed using the variations of $\mathscr{M}$ and $\mathscr{M}_{1}$.

Note that the equation 4.1 can be used to compute the inverse of $D^{\left(N_{5}\right)}$. In this case the linear system to be solved is:

$$
D^{\left(N_{5}\right)} x=b
$$


Let $y=\left(x, y_{2} \ldots, y_{N_{5}}\right)$ and $z=(b, 0 \ldots, 0)$ be 5-dimensional vectors. Then, from eq. 4.1 (see Appendix) we can write:

$$
\mathscr{T} y=\mathscr{T}_{1} z
$$

Using eq. 6.1 we get:

$$
\mathscr{M} P y=\mathscr{M}_{1} P z
$$

In this way, one can use the usual solver for the 5-dimensional matrix and get the solution $x$ of the 4-dimensional system 4.2 form the first block-component of $y$.

\section{Conclusion}

In this paper we have given an explicit calculation of the effective pseudofermion action that is used in dynamical domain wall simulations. We note that the extra pseudofermion terms may cause the current simulation algorithms to produce gauge fields in the region of the Aoki phase.

To cure this phenomenon we have proposed a simple solution which can be easily implemented using the algebraic relations between overlap and domain wall fermions.

\section{References}

[1] D.B. Kaplan, A Method for Simulating Chiral Fermions on the Lattice Phys. Lett. B 228 (1992) 342.

[2] R. Narayanan, H. Neuberger, Infinitely many regulator fields for chiral fermions, Phys. Lett. B 302 (1993) 62, A construction of lattice chiral gauge theories, Nucl. Phys. B 443 (1995) 305.

[3] V. Furman, Y. Shamir, Axial symmetries in lattice QCD with Kaplan fermions, Nucl. Phys. B439 (1995) 54-78

[4] A. Boriçi, Truncated Overlap Fermions: the link between Overlap and Domain Wall Fermions, in V. Mitrjushkin and G. Schierholz (edts.), Lattice Fermions and Structure of the Vacuum, Kluwer Academic Publishers, 2000.

[5] A. Boriçi, Computational methods for the fermion determinant and the link between overlap and domain wall fermions, in QCD and Numerical Analysis III, ed. Boriçi et al, Springer 2005.

[6] H. Aoki et al, Lattice QCD with two dynamical flavors of domain wall quarks, hep-lat/0411006

[7] A. Boriçi, Truncated Overlap Fermions, Nucl. Phys. Proc. Suppl. 83 (2000) 771-773

[8] R.C. Brower, H. Neff, K. Orginos, Mobius Fermions: Improved Domain Wall Chiral Fermions, hep-lat/0409118

[9] H. Neuberger, Exactly massless quarks on the lattice, Phys. Lett. B 417 (1998) 141 


\section{Appendix: explicit calculation of $S_{\mathbf{P F}}$}

Multiplying $\mathscr{M}$ from the right by $P$ we obtain:

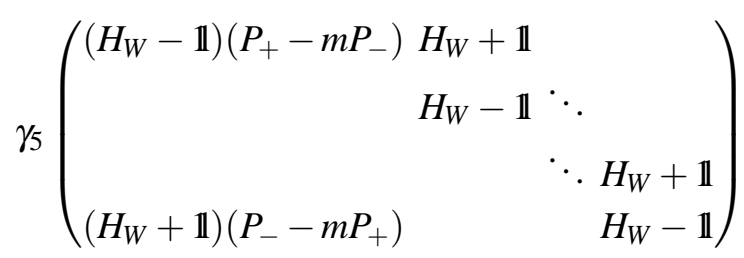

Further, multiplying this result from the left by the inverse of the diagonal matrix:

$$
H_{5}=\gamma_{5} \operatorname{diag}\left(H_{W}-\mathbb{1}, \ldots, H_{W}-\mathbb{1}\right)
$$

we get:

$$
\mathscr{T}=H_{5}^{-1} \mathscr{M} P
$$

Let $X_{i j}, i, j=1, \ldots, N_{5}$ be the four dimensional blocks of the inverse of $\mathscr{T}$. Then a straightforward calculation gives:

$$
X_{1 j}=\frac{\mathbb{1}}{\left(P_{+}-m P_{-}\right)-T^{N_{5}}\left(P_{-}-m P_{+}\right)} T^{j-1}=\frac{\mathbb{1}}{D^{\left(N_{5}\right)}} \gamma_{5} \frac{\mathbb{1}}{1+T^{N_{5}}} T^{j-1}
$$

for $j=1, \ldots, N_{5}$ and

$$
X_{i 1}=T^{N_{5}-i+1}\left(P_{-}-m P_{+}\right) X_{11}
$$

for $i=2, \ldots, N_{5}$ and

$$
X_{i j}=T^{N_{5}-i+1}\left(P_{-}-m P_{+}\right) X_{1 j}+\sum_{k=0}^{N_{5}-i} \delta_{i+k, j} T^{k}
$$

for $i=2, \ldots, N_{5}, j=2, \ldots, N_{5}$. 